\title{
Outcome of Infected Nonunion of Long Bones Treated by Single- Stage Bone Grafting and External Fixation at Northwest General Hospital, Peshawar, Pakistan
}

\author{
Waqas Raza ${ }^{1}$, Raja Irfan Qadir², Shabir Awan ${ }^{3}$, Muhammad Abu Bakar ${ }^{4}$ \\ ${ }^{1}$ Assistant Professor, Department of Orthopedics, Northwest General Hospital and Research Center, Peshawar, Pakistan \\ ${ }^{2}$ Professor, Department of Orthopedics, Northwest General Hospital and Research Center, Peshawar, Pakistan \\ ${ }^{3}$ Consultant, Department of Orthopedics, Northwest General Hospital and Research Center, Peshawar, Pakistan \\ ${ }^{4}$ Specialist, Department of Orthopedics, Northwest General Hospital and Research Center, Peshawar, Pakistan
}

ABSTRACT

Background: Infected nonunion of long bones after fractures is one of the most challenging complication to treat in clinical practice. It is commonly treated by a two-stage approach; controlling infection initially and then application of external fixator and bone graft. Treatment with Ilizarov had been the gold standard but is associated with few complications. The objective of the study was to evaluate the clinical, radiological and functional outcomes of infected non-union of long bones treated with single-stage bone grafting and external fixation.

Material and Methods: This retrospective study was carried out at Orthopedics Department of Northwest General Hospital, Peshawar Pakistan from 2014 to 2019. Medical records of 17 patients, aged 32 years and treated with Singlestage bone grafting and external fixation were reviewed. Nonunion included 7 humerii, 5 femurs, 3 tibias and 2 radius/ulna. Preoperative plain radiographs for any sequestrum or sclerosed bone margins and baseline investigations (like CBC, ESR, CRP and Vitamin-D status) were reviewed. All patients underwent radical debridement, reaming of intramedullary canals, external fixation and autogenous bone grafting. The outcomes evaluated were union of the bone (clinically and radiologically), resolution of infection, complication rate and number of re-intervention surgeries.

Results: Among 17 patients, 10 were males and 7 were females. After treatment, mean follow-up duration was $9.41 \pm 4.48$ months (4-18 months). None of the patients were lost to follow-up, had recurrence of infection or required further surgery. The mean duration of bone union was $8.4 \pm 4.48$ months (range 3 to 17 months) and all of the patients achieved infection free union with satisfactory functional outcome.

Conclusions: Single-stage bone grafting and external fixation is an effective technique in terms of resolution of infection and satisfactory bone union without any complications and can be used as an alternative to llizarov for treating cases of infected nonunion of long bones.

Key words: External fixation, Infected nonunion, Long bones, Single-stage bone grafting.

Authors' Contribution:
${ }^{1}$ Conception; Literature research;
manuscript design and drafting; ${ }^{2-4}$ Critical
analysis and manuscript review; Data
analysis; Manuscript Editing.

Correspondence:

Waqas Raza

Email: mudassirazahid@gmail.com
Article info:

Received: August 20, 2020

Accepted: June 15, 2021

Cite this article. Raza W, Qadir R I, Awan S, Bakar M A. Outcome of Infected Nonunion of Long Bones Treated by Single-stage Bone Grafting and External Fixation at Northwest General Hospital, Peshawar, Pakistan. J Islamabad Med Dental Coll. 2021; 10(2): 76-82. Doi:

Funding Source: Nil Conflict of Interest: Nil 


\section{Introduction}

Infected nonunion of bone is an inability of a fracture to heal for 6 to 8 months with persistence of infection. ${ }^{1,2}$ Such nonunions are common in fractures caused by high-energy trauma, open fractures or as a post-operative complication of internal fixation of fractures. ${ }^{3}$ Although not very common, this condition is quite difficult to treat and poses a great challenge to Orthopedic surgeons due to various complications such as deformity, limb length discrepancy, joint stiffness, osteopenia, skin sinuses, resistance to antibiotics, bone and soft tissue defects. ${ }^{4}$ These complications exert a significant burden on the social, financial, physical and psychological aspect of the patient's wellbeing. 5,6

Multiple surgical methods are being used for treatment of infected nonunions worldwide such as bone grafting, ${ }^{5}$ tissue transfer, ${ }^{6}$ antibiotic loaded cement, ${ }^{1}$ external fixation and Ilizarov. ${ }^{7}$ Bone grafting is a common technique but is associated with certain limitations/complications while treating larger bone defects, prolonged graft incorporation time and donor site morbidity. ${ }^{8}$ Free tissue transfer is another feasible option for large bone defects and soft tissue loss but it is technically demanding and is associated with stress fractures and nonunion. ${ }^{9}$ Antibiotic cement is also associated with certain limitations as it is only suitable for small defects and a second procedure of bone grafting is usually required to achieve bone union. ${ }^{1}$

The single-stage treatment with thorough debridement and irrigation, external fixation, and autogenous cancellous bone grafting is known to be an effective technique because of shorter hospital stay, reduced cost and less chances of reintervention surgeries. This technique has minimal complications as compared to the two-stage surgical management which is associated with increased chances of recurrence of infection and nonunion.

The objective of this study was to assess the clinical, radiological (i.e., bone union, infection control, deformity and limb length discrepancy) and functional outcomes (i.e., limp, joint stiffness, reflex sympathetic dystrophy or pain) of infected nonunion of long bones treated with single-stage bone grafting and external fixation.

\section{Material and Methods}

This retrospective study was carried out at the Orthopedics Department of Northwest General Hospital, Peshawar Pakistan from 2014 to 2019 after being approved by the Ethics Committee of Northwest General Hospital. Patients $(n=17)$ above 18 years of age, of both genders with infected nonunion of long bones treated at our institute were included after obtaining written informed consent for the use of clinical details and images. Since it is an uncommon presentation in our setting, hence only 17 cases could be evaluated for the study. No specific exclusion criteria were used as a smaller number of patients was anticipated. All the patients had active infection with purulent discharge through sinuses. Antibiotics were stopped 72 hours before surgery. Outcomes were evaluated by a multidisciplinary team including orthopedic surgeons, radiologists and infectious disease specialists.

During surgery, standard incisions were given with patients placed in supine position, followed by radical debridement of the infected avascular tissue including skin, subcutaneous tissue, fascia, muscles, fibrous tissue and necrotic bone. Paprika sign (active punctate bleeding bone) was taken as an indication of vital bone and proximal and distal medullary 
canals were curetted and reamed to remove all necrotic debris. Bone and soft tissue cultures were taken for culture and antibiotic sensitivity (C/S). External fixation of the long bones was then done under image intensifier using Schanz screws in unilateral uniplanar mode.

Cancellous bone graft, harvested from patient's iliac crest was then applied at freshened bone ends. Empirical antibiotics were given to all patients intravenously before culture and sensitivity report. The wounds were closed in layers with suction drain in place.

Postoperatively, all patients were given parenteral antibiotics according to their culture and sensitivity throughout their hospital stay and were discharged with enteral antibiotics for 6 weeks. C/S report of seven patients showed MRSA while growth of Staphylococcus aureus, Proteus mirabilis, Serratia species and Pseudomonas aeruginosa was seen in two patients, each. Sample of one of the patients showed Edwardsiella while another one had Enterobacter infection. All MRSA patients were sensitive to linezolid, which was continued orally for 6 weeks.

Drains were removed on first or second postoperative day. Patients were advised active assisted range of motion exercises for joints and isometric muscles exercises on second postoperative day. Radiographs were taken and reviewed on first postoperative day and then monthly until union was achieved. The fixator was removed when radiographs showed solid union. The cost of all procedures including debridement, bone grafting and external fixation were borne by the patients.

Mean values were calculated and Kruskal-Wallis test was applied to determine the intergroup differences in terms of bone and time to complete union. The test statistic was adjusted for ties. Multiple comparisons were performed if the test showed significant difference across samples. $P$-value less than .05 was considered as statistically significant.

\section{Results}

Out of 17 patients included in the study, 10 were males and 7 were females with a mean age of 31.76 \pm 7.59 years (age range 18-45 years). Long bones with infected nonunion included humerus $(n=7)$, femur $(n=5)$, tibia $(n=3)$ and radius/ulnas $(n=2)$, respectively (Figure 1 ). The previous procedures included plating (12 patients), interlocking nail (3 patients), rush nail (1 patients), and screw \& K wires (1 patient). Intraoperative specimen of all the patients were positive for bacterial growth. The patients were followed up for a mean duration of $9.41 \pm 4.48$ months (range 4 to 18 months) after the surgery and none of the patient was lost to followup.

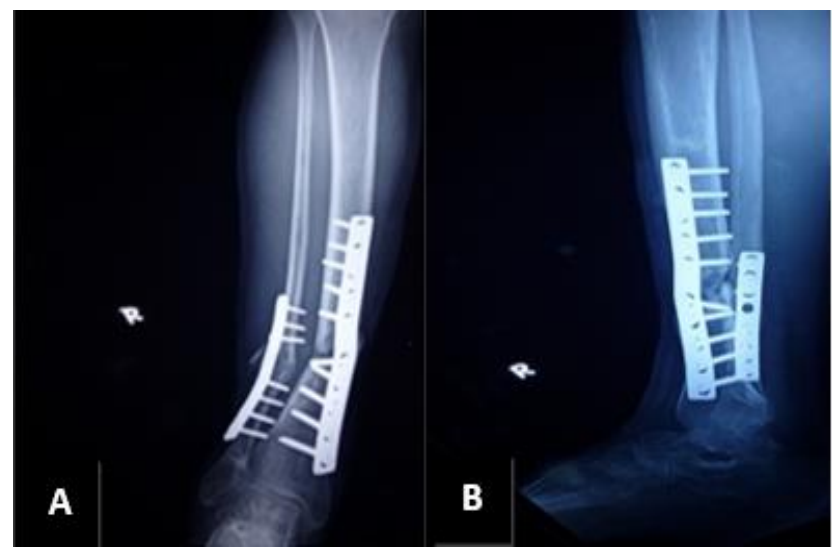

Figure 1: Infected nonunion of tibia with broken implant in situ. A: AP view, B: Lateral view.

Satisfactory bone union without infection was achieved in all patients (Figures $2 \& 3$ ). There was statistically no significant difference in recovery gender wise. No bony complications such as deformity or limb length discrepancy were recorded. Functional outcome was satisfactory in all patients with no limp, joint stiffness, reflex sympathetic dystrophy or pain (Figure 4). 


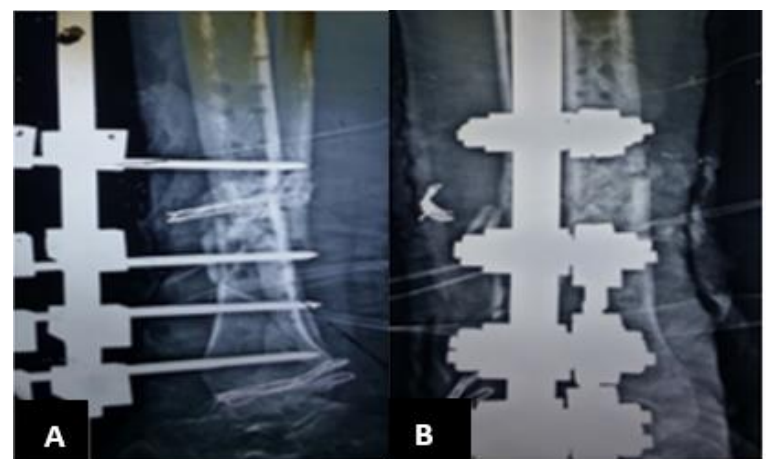

Figure 2: Postoperative view of tibia after single-stage external fixation and bone grafting. A: AP view.

B: Lateral view.
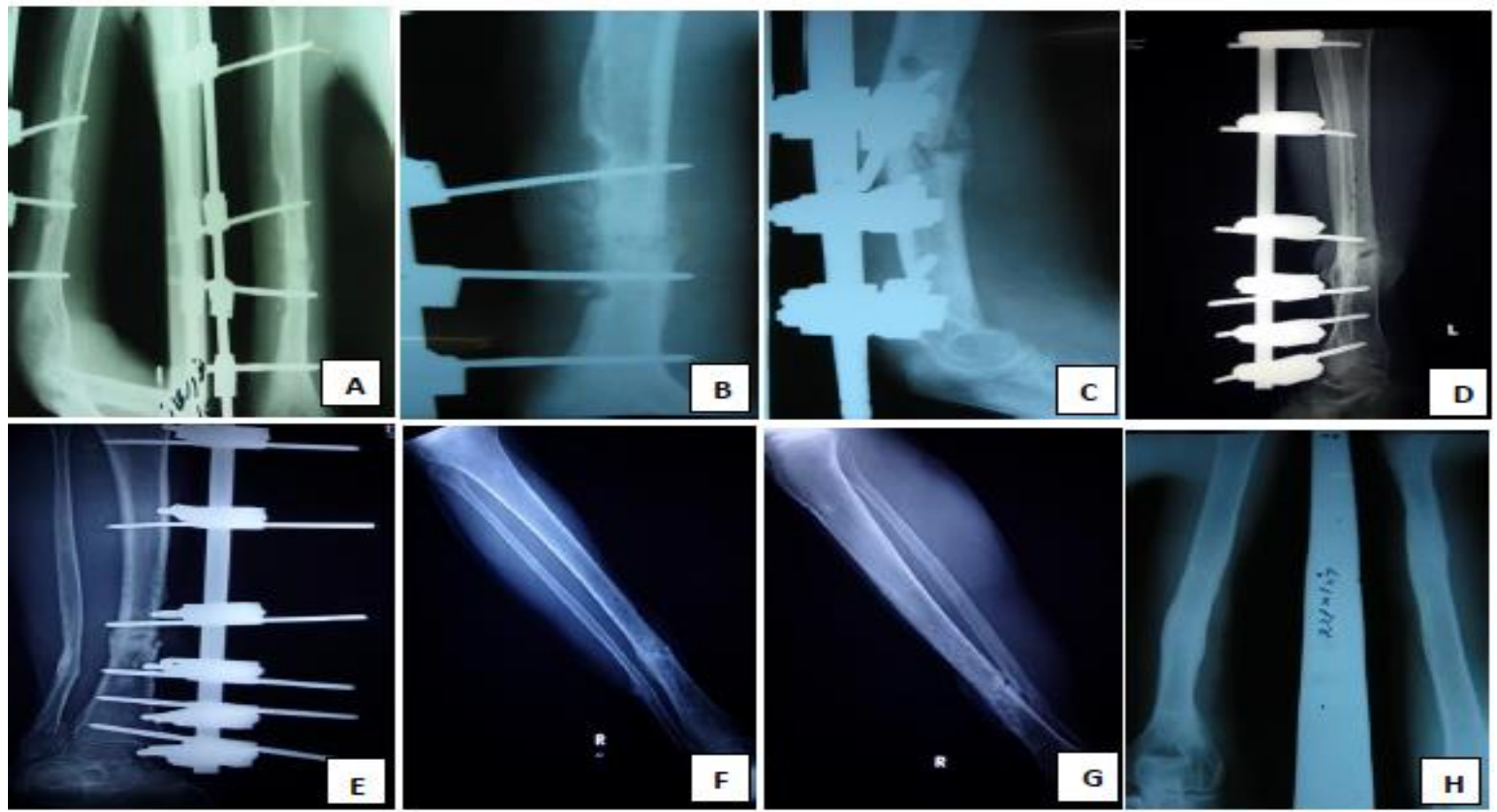

Figure 3: Postoperative follow up x-rays after single-stage external fixation and bone grafting. A, B \& C: AP and lateral view of humerus at 5 months. D: X-ray at seven months (Lateral view tibia). E: X-ray at seven months (AP view tibia). $\mathrm{F}$ : X-ray at nine months (AP view tibia). G \& H: X-rays at nine months showing fracture is united.

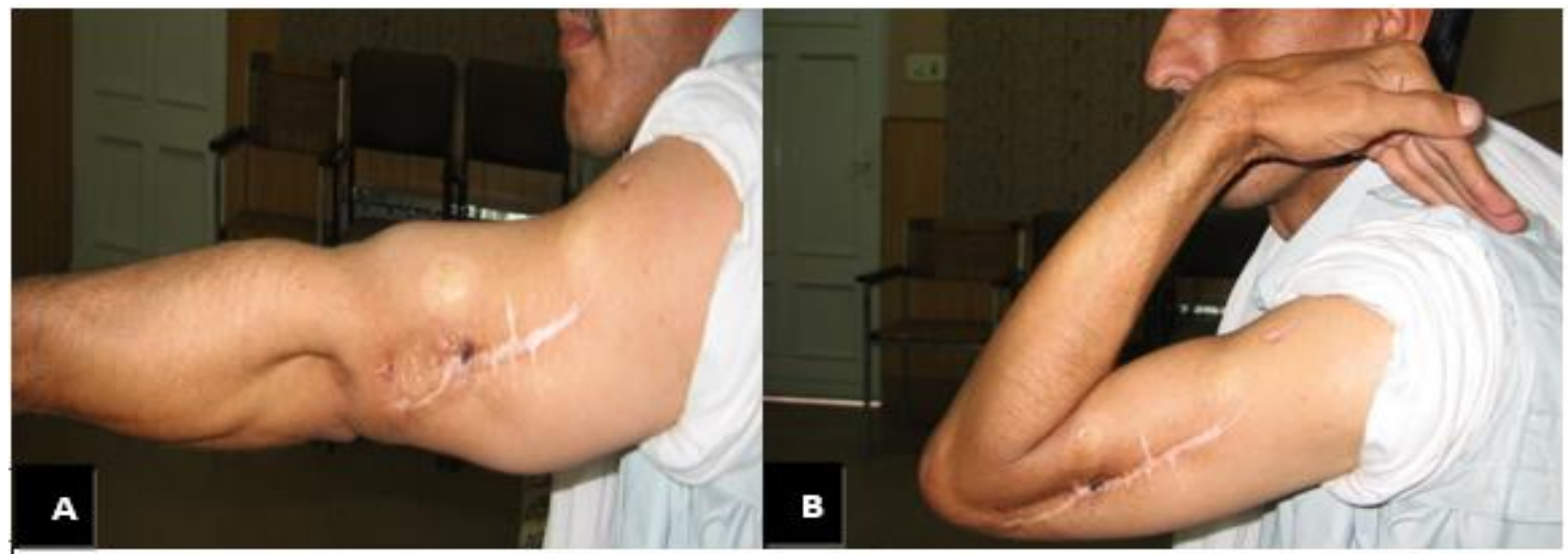

Figure 4 A \& B: Functional range of motion after union is achieved. 


\section{Discussion}

This study aimed to evaluate the effectiveness of single-stage bone grafting and external fixation for treatment of infected nonunion of long bones. Favorable outcomes were observed in terms of resolution of infection, complete union and regain of functional mobility. Infected nonunion of long bones is one of the most difficult problems to treat in orthopedics practice. ${ }^{10}$ It is difficult to control the infection and achieve union ${ }^{1,2}$ and thus the management is challenging for both the patient and the treating surgeon. ${ }^{1,3,4}$ There are two main protocols for the management of infected nonunion; "One-stage" protocol involves simultaneous clearance of infection by radical debridement and bone stabilization with external fixation and a "two-stage" protocol wherein infection is eradicated in the first stage followed by bone stabilization with internal or external fixation. Conventional strategy for infected nonunion is the two-stage technique.

In the past decade llizarov has been considered as the gold standard treatment for infected nonunion ${ }^{2}$ but it has a number of complications. ${ }^{11}$ However, this method increases hospital stay and is costly. Many authors have reported success by treatment with antibiotic impregnated intramedullary nailing but this technique can lead to infection of entire medullary canal of the bone. ${ }^{11}$ Furthermore, in locally infected cases some authors have reported good success rate with plating and bone graft also. ${ }^{12}$

Bone grafting plays a critical role in promoting bone healing in infected non-union. ${ }^{13-15}$ External fixation has been proven preferable in infected nonunion for reasons like fixation away from infected area, easy to apply and reduced risk of infection recurrence. ${ }^{16,17}$

One-stage treatment has resulted in avoidance of multiple surgeries and anesthesia as well as reduced cost and hospital stay. Emara et al. also confirmed that one stage strategy had lower risk of recurrence of infection. ${ }^{17}$ Lei and $\mathrm{Yi}$ treated 19 patients of infected non-union of femur utilizing Single-stage protocol with external fixation or bone grafting and achieved infection-free union in all. ${ }^{18}$ Their results are similar to our study using single-stage protocol with external fixation.

Some of the advantages of one-stage bone grafting and external fixation as observed in this study are a shorter hospital stay, cost-effectiveness, reduced time to bone healing and safer and predictable achievement of infection free union. Study by Liu et al. ${ }^{19}$ showed feasibility of one-stage open cancellous bone grafting in the treatment of infected nonunions in a rabbit model. Many surgeons have also confirmed that satisfactory results can be achieved with one-stage procedure, including a shorter hospital stay. ${ }^{20-22}$ Treatment of infected non-union by open bone grafting combined with external fixation is challenging because multiple dressings are used for wound coverage, ${ }^{14,23,24}$ thereby increasing time of bone union and wound healing. This leads to increased cost and hospital stay. We however report external fixation as a cost-effective procedure. Regarding reduced time to bone healing, Lei and $\mathrm{Yi}$ also reported that all patients had achieved bony union with resolution of infection using single-stage protocol. Their study had average follow-up of 3.5 years, hence longer time to achieve bone healing as compared to our study. ${ }^{18}$ Bose et al. found that eradication of infection was more predictably achieved by the single surgical procedure which has also been reported by other authors. ${ }^{24,25}$ Also, in infected non unions external fixation is preferred and safer method than internal fixation. ${ }^{26}$

In this study, infection free union has been achieved in all patients, which is almost similar to the average data recorded in the above-mentioned studies. In order to increase the success rates, we believe that 
preparing patients for surgery keeping in view their specific conditions, good patient's compliance and meticulous post-operative care is necessary. Favorable results of our study suggest that onestage bone grafting and external fixation may be a feasible alternative treatment option.

The main limitation of our study is a small sample size, no comparison group, and absence of functional outcome scores. However, using our single-stage protocol we achieved infection free union in all cases and majority of our patients had attained full range of motion. Moreover, the study lacks a direct comparison with any other treatment options and thus further randomized controlled trials are needed to draw a more valuable conclusion.

\section{Conclusion}

This study suggested that single-stage technique is effective in treating infected nonunion with complete resolution of infection and bone union. The treatment with external fixation and one stage bone grafting can be used as alternative method of fixation for obtaining fracture union with a good functional and bone results.

\section{References}

1. Yin P, Zhang L, Li T, Zhang L, Wang G, Li J, et al. Infected nonunion of tibia and femur treated by bone transport. JOSR. 2015; 10(1):1-9. Doi: 10.1186/s13018-015-0189-5.

2. Shahid $M$, Hussain A, Bridgeman $P$, Bose D. Clinical outcomes of the llizarov method after an infected tibial non-union. Arch Trauma Res. 2013; 2(2):71. Doi: 10.5812/atr.11300.

3. Harshwal RK, Sankhala SS, Jalan D. Management of nonunion of lower-extremity long bones using monolateral external fixator-report of 37 cases. Injury. 2014; 45(3):560-7. Doi: 10.1016/j.injury.2013.11. 019.

4. Deng Z, Cai L, Jin W, Ping A, Wei R. One-stage reconstruction with open bone grafting and vacuumassisted closure for infected tibial non-union. AMS. 2014; 10(4):764-72. Doi: 10.5114/aoms.2013.34411.
5. Ozaksar K, Sugun T, Toros T, Gurbuz $Y$, Kayalar M, Ozerkan F. Free vascularized fibular grafts in Type 3 open tibia fractures. AOTT. 2012; 46(6): 430-37. Doi:10.3944/aott.v46i6.6049.

6. Yin $P$, Zhang $Q$, Mao Z, Li T, Zhang L, Tang P. The treatment of infected tibial nonunion by bone transport using the llizarov external fixator and a systematic review of infected tibial nonunion treated by Ilizarov methods. Acta Orthop Belg. 2014; 80(3): 426-35.

7. Naidu, KV Dhanwantary. Efficacy external fixator in the management of infected nonunion fractures. IJOS. 2018; 4(1): 164-66. Doi: 10.22271/ortho. 2018.v4.i1c.25.

8. Sun $Y$, Zhang $C$, Jin $D$, Sheng J, Cheng $X$, Zeng $B$. Treatment for large skeletal defects by free vascularized fibular graft combined with locking plate. Arch Orthop Trauma Surg. 2010; 130(4): 473-9. Doi: 10.1007/s00402-009-0898-5.

9. Chaudhary MM. Infected nonunion of tibia. Indian J Orthop. 2017; 51(3): 256-68. Doi: 10.4103/ortho. IJOrtho_199_16.

10. Park J, Yang KH. Indications and outcomes of augmentation plating with decortication and autogenous bone grafting for femoral shaft nonunions. Injury. 2013; 44(12): 1820-5. Doi: 10.1016/j.injury.2013.02.021.

11. Liu Y, Yushan M, Liu Z, Liu J, Ma C, Yusufu A. Complications of bone transport technique using the Ilizarov method in the lower extremity: a retrospective analysis of 282 cases over 10 years. BMC Musculoskelet Disord. 2020; 21: 354. Doi: 10.1186/s12891-020-03335-w.

12. Archdeacon MT, Messerschmitt P. Modern papineau technique with vacuum-assisted closure. J Orthop Trauma. 2006; 20(2): 134-7. Doi: 10.1097/01.bot. $0000184147.82824 .7 \mathrm{c}$

13. Saleh M, Kreibich DN, Ribbans WJ. Circular frames in the management of infected tibial non-union: a modification of the Papineau technique. Injury. 1996; 27(1): 31-3. Doi: 10.1016/0020-1383(95)00164-6.

14. Tulner SA, Schaap GR, Strackee SD, Besselaar PP, Luitse JS, Marti RK. Long-term results of multiplestage treatment for posttraumatic osteomyelitis of the tibia. J Trauma Acute Care Surg. 2004; 56(3): 63342. Doi: 10.1097/01.TA.0000112327.50235.0A.

15. Ueng WN, Shih $\mathrm{CH}$. Semiopen cancellous bone grafting. A 2-step method for closing small infected tibial bone defects. Clin Orthop Relat Res. 1994; 306: 175-82. PMID: 8070192.

16. Petty WI, Spanier SU, Shuster JJ, Silverthorne CA. The influence of skeletal implants on incidence of infection. Experiments in a canine model. J Bone Joint Surg. 1985; 67(8): 1236-44. PMID: 3902846. 
17. Emara KM, Ghafar KE, Diab RA. One stage versus two stages strategies in the management of femoral shaft infected nonunion. MOJ Sports Med. 2017; 1(2): 246.

18. Lei $H$, Yi L. One-stage open cancellous bone grafting of infected fracture and nonunion. J Orthop Surg. 1998; 3(6): 318-23. Doi: 10.1007/s007760050059.

19. Liu DQ, Zhang BS, Liu HB. Experimental observation of open cancellous bone graft in treatment of infected fracture nonunion. Chin J Orthop Trauma. 2004; 6: 1370-3.

20. Chen Z-W, Liu H, Zhai W-L, Zeng J-H. Treatment of infected bone defect with one stage open cancellous bone grafting. Zhongguo Gu Shang (CJOT). 2008; 21(5): 377-8. PMID: 19108471.

21. Lu WJ, Qian HB, Li B. Treatment of chronic osteomyelitis by modified I-stage open cancellous bone grafting post debridement. Chin J Trauma. 2004; 20: 288-90.
22. Papineau LJ. Excision-graft with deliberately delayed closing in chronic osteomyelitis. La Nouvelle Presse Medicale. 1973; 2(41): 2753-5. PMID: 4589881.

23. Sachs BL, Shaffer JW. A staged Papineau protocol for chronic osteomyelitis. Clin Orthop Relat Res. 1984; 184: 256-63. PMID: 6368084.

24. Bose D, Kugan R, Stubbs D, McNally M. Management of infected nonunion of the long bones by a multidisciplinary team. Bone Joint J. 2015; 97(6): 8147. Doi: 10.1302/0301-620X.97B6.33276.

25. Raj M, Singh J, Mukhopadhya J, Gill SP, Sheopaltan $\mathrm{SK}$, Kumar $\mathrm{H}$, et al. Evaluation of Single-stage protocol using Locked plate as primary fixation in treatment of infected non-union of long bones. IJOS. 2016; 2(4): 86-90.

26. Thakeb MF. Compression distraction for the management of complex femoral nonunion. Egypt Orthop J. 2017; 52(2): 91. Doi: 10.4103/eoj.eoj_ 24_17. 